EPJ manuscript No.

(will be inserted by the editor)

\title{
Static quark anti-quark free and internal energy in 2-flavor QCD
}

\author{
Olaf Kaczmarek $^{1}$ and Felix Zantow ${ }^{2}$ \\ 1 Fakultät für Physik, Universität Bielefeld, D-33615 Bielefeld, Germany \\ ' 2 Physics Department, Brookhaven National Laboratory, Upton, NY 11973 USA
}

May 7, 2018

\begin{abstract}
We study the change in free and internal energy due to the presence of a heavy quark anti-quark pair in a thermal heat bath in QCD with 2-flavors of staggered quarks at finite temperature. We discuss string breaking below as well as screening above the transition. Similarities and differences to the quenched case are discussed.
\end{abstract}

PACS. 11.15.Ha, 11.10.Wx, 12.38.Mh, 25.75.Nq

\section{Introduction}

The study of the fundamental forces between quarks is an essential key to the understanding of QCD and the occurrence of different phases at high temperatures $(T)$ and/or non-zero quark chemical potential $(\mu)$. The free energy of a static quark anti-quark pair [1, separated by distance $r$, is an important tool to analyze the $r$ and $T$ dependence of the QCD forces. Similar to the free energies also the internal energies have been introduced [2,3] and are expected to play an important role in the discussion of quarkonia binding properties [4,5,6]. The structure of ' these observables in the short and intermediate distance regime, $r T \lesssim 1$, is relevant for the discussion of in-medium ' modifications of heavy quark bound states [7, 8, 9, 10,11] which are sensitive to thermal modifications of the heavy quark potential 12. Up to quite recently [13.14, however, most of these discussions concerned quenched QCD.

Several qualitative differences, however, are to be expected when taking the influence of dynamical fermions into account; the phase transition in QCD will appear as an crossover rather than a 'true' phase transition with related singularities in thermodynamic observables. Moreover, in contrast to a steadily increasing confinement interaction in quenched QCD, in full QCD the strong interaction below $T_{c}$ will show a qualitative different behavior due to the possibility of string breaking. Thus it is quite important to extend our developed concepts for the analysis of free energies and internal energies in quenched QCD 2 15, 16] to the complex case of QCD. This has recently been done for 2- and 3-flavor QCD [13,17, 18].

\section{Quark anti-quark free energy}

We will discuss here lattice results for the quark anti-quark free and internal energies in 2-flavor QCD $\left(N_{f}=2\right)$ using an improved staggered fermion action with quark mass $m / T=0.4$. Any further detail on this study can be found in 13,17,18. For information on the improved actions used in these simulations see 19 20.21. While in earlier studies of the heavy quark free energy in quenched [22] and full QCD [23] the color averaged operators were analyzed we discuss here the free and also the internal energies in the color singlet channel. The static quark sources are described by the Polyakov loop, $W(\boldsymbol{x})=\prod_{\tau=1}^{N_{\tau}} U_{0}(\boldsymbol{x}, \tau)$ with $U_{0}(\boldsymbol{x}, \tau) \in S U(3)$ being defined on the lattice link in time direction. The free energies in the color singlet channel is then given by [1,24]

$$
e^{-F_{1}(r) / T+C}=\frac{1}{3} \operatorname{Tr}\left\langle W(\boldsymbol{x}) W^{\dagger}(0)\right\rangle,
$$

where $r=|\boldsymbol{x}|$ and $C$ is a suitably chosen renormalization constant. The operator used here to calculate $F_{1}(r, T)$ is not gauge invariant. Our calculations thus have been performed in Coulomb gauge [25] .

\subsection{Renormalized free energy}

As noted above, the quark anti-quark free energy (11) and the expectation value of the Polyakov loop suffer from divergences and require renormalization. We follow here the conceptual approach recently suggested for the quark antiquark free energies in quenched QCD [2] and apply it to our lattice results in full QCD. First experiences with this renormalization prescription in QCD are reported in [13. 14. Renormalization prescriptions for the Polyakov loop expectation value are discussed in [28,29].

At distances much smaller than the inverse temperature, $r \ll 1 / T$, the dominant scale is set by $r$ and the running coupling will be controlled by this scale, $r \ll 1 / \Lambda_{Q C D}$ 26. 16. In this limit the free energies are dominated by 


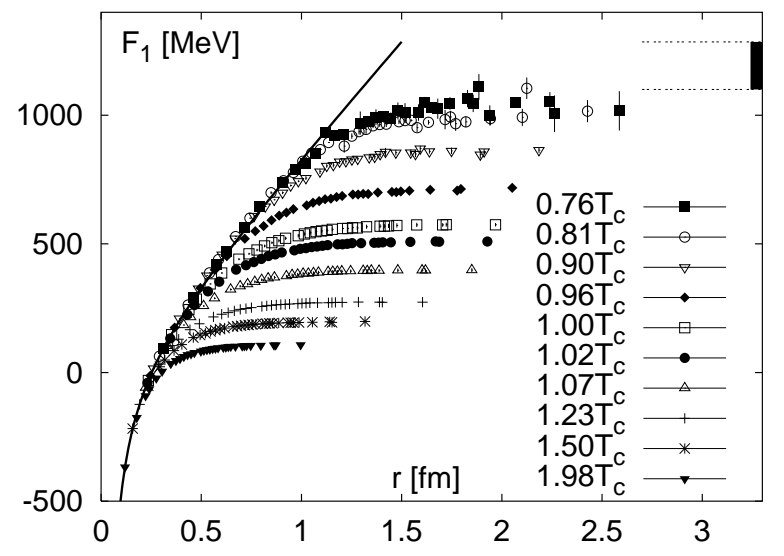

Fig. 1. Results for the renormalized color singlet free energies calculated in 2-flavor QCD for $0.75<T<2.00$. The solid line represents the heavy quark potential at zero temperature, $V(r)$, given in 26]. At $T=0$ string breaking is expected at distances about $1.3-1.5 \mathrm{fm}$ [27. The corresponding energies, $V(1.3-1.5 \mathrm{fm}) \simeq 1100-1285 \mathrm{MeV}$, are shown by the band.

one-gluon exchange, i.e. are given by the heavy quark potential,

$$
F_{1}(r, T) \equiv V(r) \simeq-\frac{4}{3} \frac{\alpha(r)}{r} .
$$

We have neglected here any constant contributions to the free energy which, in particular, will dominate the large distance behavior of the free energy. Moreover, we already anticipated here the running of the coupling with the dominant scale $r$. Following 2] we can use this property to fix the constant $C$ in (11) by matching $F_{1}(r, T)$ to the zero temperature heavy quark potential at small distance, $F_{1}(r \ll 1 / T, T) \simeq V(r)$. Once the free energy is fixed at small distances also the large distance behavior is fixed as no additional divergences get introduced at finite temperature.

Our lattice results for $F_{1}(r, T)$ calculated in 2-flavor QCD are summarized in Fig. 1 at several temperatures in the vicinity of the phase transition. Due to renormalization the free energies coincide with the heavy quark potential, $V(r)$, at small distances $(V(r)$ is specified in 17. [18,26]). When going to larger distances, however, thermal modifications become important and the different effects from color screening $\left(T \gtrsim T_{c}\right)$ and string breaking $\left(T \lesssim T_{c}\right)$ can be studied.

\subsection{Color screening and string breaking}

As the free energies shown in Fig. 1 rapidly change from the zero temperature like behavior at small distances to an almost constant behavior at large distances we introduce here scales that may characterize this qualitative change. For this purpose we introduced in 2 a scale $r_{\text {med }}$ defined through $F_{\infty}(T)=V\left(r_{m e d}\right)$, where $F_{\infty}(T)$ is the plateau value which the free energy approaches at large

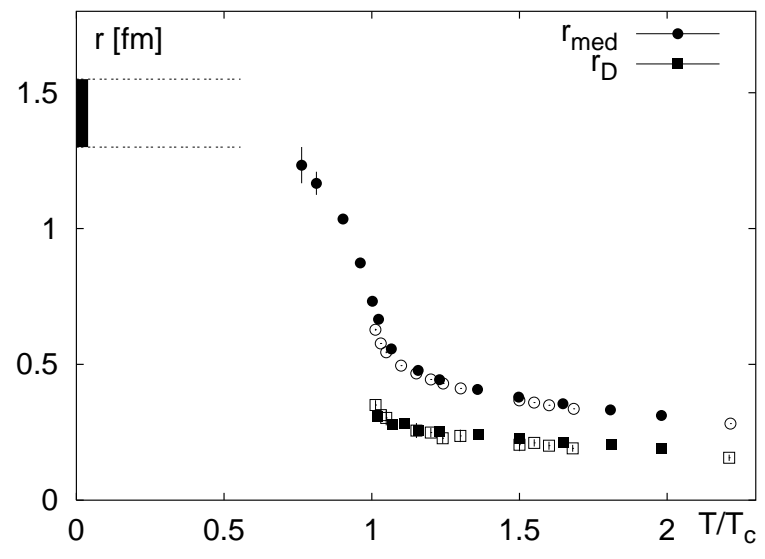

Fig. 2. The Debye screening radius, $r_{D}(T)$, and the scale $r_{\text {med }}(T)$ as function of temperature in physical units obtained from 2-flavor QCD lattice studies (filled symbols). The open symbols correspond to calculations in quenched QCD [2,16]. Further analysis of these data with respect to quarkonium dissociation temperatures can be found in 30.

distances, $F_{\infty}(T) \equiv \lim _{r \rightarrow \infty} F_{1}(r, T)$. This scale characterizes the typical distance at which string breaking and color screening become relevant. Alternatively one can at high temperature characterize screening properties of the QCD plasma $\left(T \gtrsim T_{c}\right.$ ) in terms of the Debye screening length, $r_{D}(T)$, which is commonly defined as the inverse of the screening mass, $r_{D}(T) \equiv 1 / m_{D}(T)$. We also estimated this scale by extracting the screening masses nonperturbatively from the exponential fall off that the free energies show above $T_{c}$ at large distances. Our results for $r_{D}\left(T \gtrsim T_{c}\right)$ and $r_{m e d}$ are shown in Fig. 20 as function of $T / T_{c}$ and are compared to findings in quenched QCD [2] 16. In general we find that both scales decrease with increasing temperature and $r_{m e d}(T) \gtrsim r_{D}(T)$. We note that both scales are expected to behave like $1 / g T$ in the high temperature limit. On the other hand, when comparing both scales to the findings in quenched QCD no or only little differences in the temperature range $1.2 \lesssim T / T_{c} \lesssim 2$ could be identified. However, at temperatures close to and below the transition, $T \lesssim 1.2 T_{c}$, differences become quite apparent. These differences signal the qualitative change in the phase transition when changing from quenched $\left(1^{\text {st }}\right.$ order transition) to full QCD (crossover). In QCD $r_{m e d}$ is finite also below $T_{c}$ due to string breaking and shows a rapid increase with decreasing temperatures ${ }^{1}$. In fact, the distance where the string is commonly expected to break at $T=0$ [27], $r_{\text {med }}(T=0) \simeq 1.3-1.5 \mathrm{fm}$, is almost approached already at $T \simeq 0.8 T_{c}$. The quark anti-quark free energies in 2-flavor QCD thus are expected to show only little deviations from $V(r)$ at $T=0$ at $T \gtrsim 0.8 T_{c}$.

This is also evident when analyzing the plateau values, $F_{\infty}(T)$, summarized in Fig. 3 as function of $T / T_{c}$ and again compared to recent findings in quenched [3] and 3-flavor QCD [14] for temperatures in the vicinity

\footnotetext{
1 In quenched QCD $F_{1}\left(r, T<T_{c}\right)$ signals strict confinement and $r_{m e d}$ is infinite.
} 


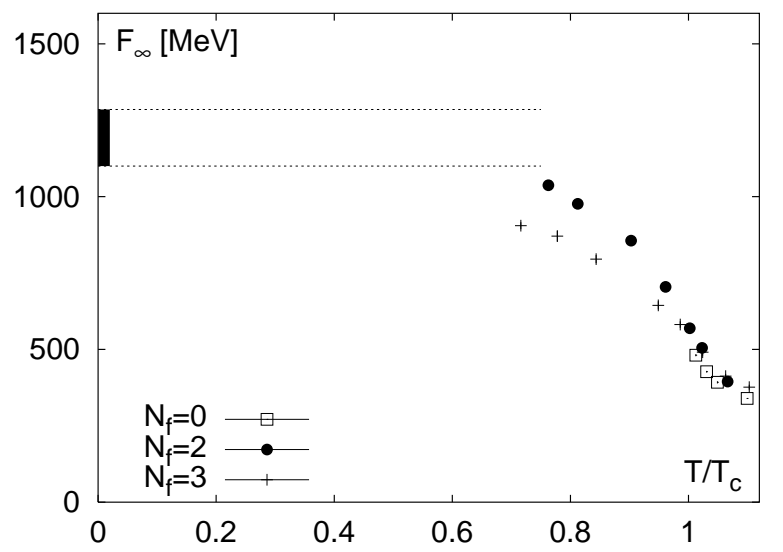

Fig. 3. The plateau value of the quark anti-quark free energy, $F_{\infty}(T)$, calculated in 2-flavor QCD as function of $T / T_{c}$ at temperatures in the vicinity and below the phase transition. The dashed band show again the string breaking energies at $T=0$, $V\left(r_{\text {break }}\right)$, with $r_{\text {break }} \simeq 1.3-1.5 \mathrm{fm}$ [27. The open symbols $\left(T \gtrsim T_{c}\right.$ ) correspond to $F_{\infty}(T)$ in quenched QCD (from Ref. [2]) and the crosses to 3-flavor QCD studies (from [14).

and below $T_{c} \cdot{ }^{2}$ At $T \simeq 0.8 T_{c} F_{\infty}(T)$ still is compatibel with the $T=0$ values, $V(r=\infty) \simeq 1.1 \mathrm{GeV}$ given in 8,27. It, however, rapidly drops in the vicinity of the transition to about half of this value, $F_{\infty}\left(T_{c}\right) \simeq 570 \mathrm{MeV}$. Although some flavor dependence can clearly be identified when comparing results in 2- and 3-flavor QCD the value at $T_{c}$ is almost the same. A similar value is also found in quenched QCD just above $T_{c}$. At very high temperatures perturbation theory [31] suggests also negative values for $F_{\infty}(T)$, i.e.

$$
F_{\infty}(T) \simeq-\frac{4}{3} m_{D}(T) \alpha(T) \simeq-\mathcal{O}\left(g^{3} T\right) .
$$

Such a behavior has indeed been observed in lattice studies of quenched QCD at high temperatures [2, 3, 32].

\section{Quark anti-quark internal energy and entropy}

It has been argued that the quark anti-quark free energy, $F_{1}(r, T)=U_{1}(r, T)-T S_{1}(r, T)$, contains non-trivial entropy contributions, i.e. $S_{1}=S_{1}(r, T)$, which, in particular, could make the analysis of thermal modifications of the finite temperature potential complicated [2]3]. We briefly discuss here the quark anti-quark internal energy ${ }^{3}$,

\footnotetext{
${ }^{2}$ The comparison of $F_{\infty}(T)$ performed here clearly depends on the relative normalization of the corresponding heavy quark potentials used for renormalization. We fixed these over all contributions such that the Cornell parameterization of $V(r)$ in all cases $\left(N_{f}=0,2,3\right)$ contains no constant contribution.

3 As we calculate the internal energy and entropy using the renormalized free energies, $U_{1}(r, T)$ and $S_{1}(r, T)$ are properly fixed by construction. However, the quark anti-quark internal
}

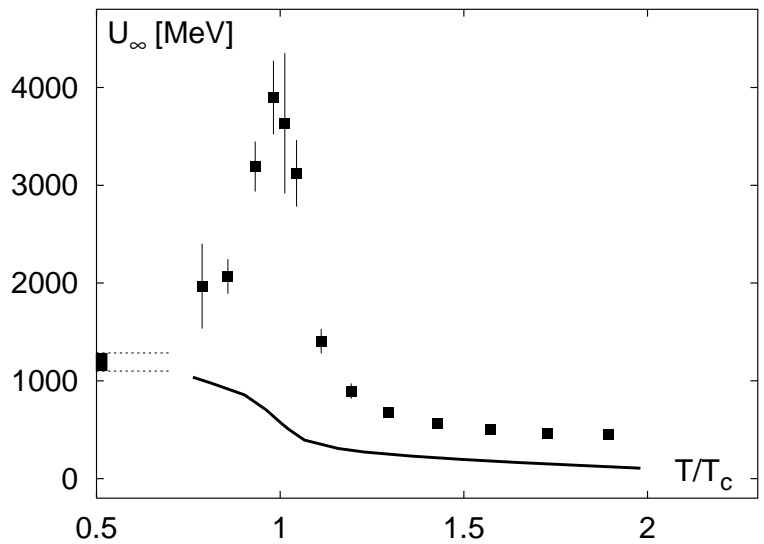

Fig. 4. The asymptotic free energy, $F_{\infty}$ (line), and the internal energy, $U_{\infty}$ (filled symbols), in 2-flavor QCD as function of $T / T_{c}$. The dashed band shows again $V(r \equiv \infty) \simeq 1100-1285$ $\mathrm{MeV} 827$.

$U_{1}(r, T)=-T^{2} \partial\left(F_{1} / T\right) / \partial T$, and entropy, $S_{1}(r, T)=$ $-\partial F_{1} / \partial T$.

It is important to realize from (3) that although the entropy contribution at (infinite) large distances, $S_{\infty}(T)$, will vanish in the perturbative high temperature limit, the quantity $T S_{\infty}(T)$ will increase and dominate the difference between free and internal energy, i.e.

$$
U_{\infty}(T)-F_{\infty}(T)=T S_{\infty}(T) \simeq+\mathcal{O}\left(g^{3} T\right) .
$$

In the limit of zero temperature, however, the observable $T S_{\infty}(T)$ is supposed to vanish as $S_{\infty}(T)$ is a dimensionless quantity. A qualitative change of the temperature dependence of the observable $T S_{\infty}(T)$ when going from $T=0$ to high temperatures, $T \rightarrow \infty$, is not obvious and if present will demonstrate the phase change from confinement to deconfinement.

Our results for $F_{\infty}(T)$ and $U_{\infty}(T)$ are summarized in Fig. [4 as function of $T / T_{c}$. The observable, $T S_{\infty}(T)=$ $U_{\infty}(T)-F_{\infty}(T)$, can easily be deduced when comparing the values for $U_{\infty}(T)$ with the values for $F_{\infty}(T)$. It can clearly be seen that $U_{\infty}(T)$ and $T S_{\infty}(T)$ exhibits a sharp peak at $T_{c}$. The internal energy falls from a value of $U_{\infty}(T) \approx 4000 \mathrm{MeV}$ at $T_{c}$ to about half of this value at a temperature of $T / T_{c} \simeq 0.8$. While the temperature dependence of $U_{\infty}(T)$ is quite strong in the vicinity of the transition and markedly different from the free energy, both quantities show a similar $T$-dependence for $1.5 \gtrsim T / T_{c} \gtrsim 2$. This is also expected at lower temperatures $\left(T \lesssim 0.7 T_{c}\right)$ were $U_{\infty}(T)$ and $F_{\infty}(T)$ are ex-

energy and entropy could also be seperately renormalized nonperturbatively at small distances, i.e. $U_{1}(r \ll 1 / T, T) \simeq V(r)$ and $S_{1}(r \ll 1 / T, T) \simeq 0$.

It is interesting to note here that in contrast to $F_{1}(r, T)$ and $U_{1}(r, T)$, the the entropy is free from any finite renormalization at $T=0$ which, in particular, could introduce flavor dependent over-all constant contributions to the finite temperature energies. 


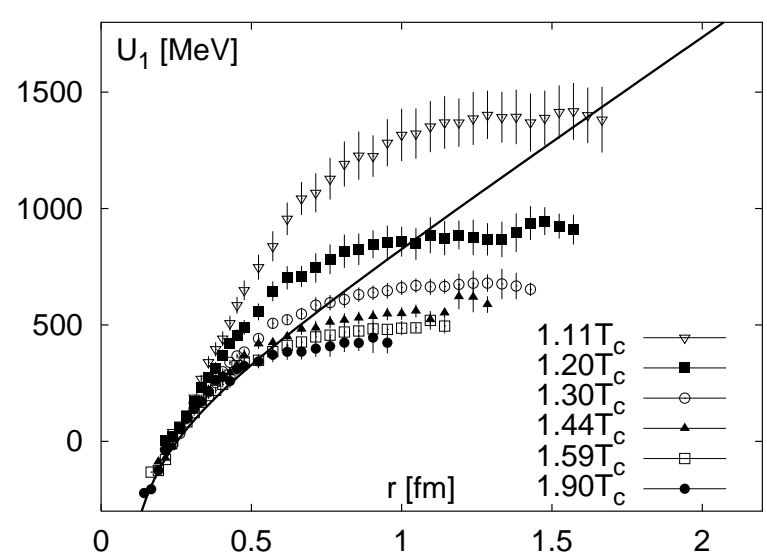

Fig. 5. The color singlet quark anti-quark internal energy above $T_{c}$. The solid line represents the $T=0$ heavy quark potential, $V(r)$.

pected to smoothly approach the same value at $T=0$, $V(r \equiv \infty) \simeq 1100-1285 \mathrm{MeV}$ (from [8,27).

Finally we show in Fig. [5 the $r$-dependence of the internal energies above $T_{c}$. Indeed $U_{1}(r, T)$ agrees with the zero temperature potential at small distances while at intermediate distances $U_{1}(r, T)$ has a steeper slope and clearly lies well above $F_{1}(r, T)$ (compare with Fig. (1). Further details on our analysis of the finite temperature energies, $F_{1}(r, T)$ and $U_{1}(r, T)$, and the entropy will be given [17, 18.

\section{Conclusions}

We have discussed thermal modifications of quark antiquark free and internal energies in the color singlet channel in QCD with dynamical quarks $\left(N_{f}=2\right)$ and compared our findings to similar studies in quenched and 3-flavor QCD. Although our comparison with the quenched case is in parts only on a qualitative level, we already leads to useful information for the study of heavy quark bound states in the plasma phase. At temperatures well above $T_{c}$, i.e. $1.2 \lesssim T / T_{c} \lesssim 2$, no or only little differences appear between results obtained in quenched and 2-flavor QCD. This might suggest that using thermal parameters extracted from energies in quenched QCD as input for model calculations is a reasonable approximation. This also supports direct investigations of heavy quarkonia in quenched lattice QCD 33/34 35. We note, however, that most of our 2-flavor QCD results differ from quenched calculations at temperatures in the vicinity and below the transition. These differences could make a discussion of possible signals for the quark gluon plasma production based on quenched QCD results complicated when temperatures close to the transition become important.

\section{Acknowledgments}

We thank the Bielefeld-Swansea collaboration for providing us their configurations with special thanks to S. Ejiri.
We would like to thank E. Laermann and F. Karsch for many fruitful discussions. F.Z. thanks P. Petreczky for his continuous support. This work has partly been supported by DFG under grant FOR 339/2-1 and by BMBF under grant No.06BI102 and partly by contract DE-AC0298CH10886 with the U.S. Department of Energy. At an early stage of this work F.Z. has been supported through a stipend of the DFG funded graduate school GRK881.

\section{References}

1. L. D. McLerran and B. Svetitsky, Phys. Rev. D24, 450 (1981).

2. O. Kaczmarek et al., Phys. Lett. B543, 41 (2002).

3. F. Zantow et al., hep-lat/0301015.

4. G. E. Brown, C.-H. Lee, and M. Rho, Nucl. Phys. A747, $530(2005)$.

5. C.-Y. Wong, hep-ph/0408020.

6. E. V. Shuryak and I. Zahed, Phys. Rev. D70, 054507 (2004).

7. N. Brambilla et al., hep-ph/0412158.

8. S. Digal, P. Petreczky, and H. Satz, Phys. Lett. B514, 57 (2001).

9. S. Digal, P. Petreczky, and H. Satz, Phys. Rev. D64, 094015 (2001).

10. C.-Y. Wong, J. Phys. G28, 2349 (2002).

11. C.-Y. Wong, Phys. Rev. C65, 034902 (2002).

12. T. Matsui and H. Satz, Phys. Lett. B178, 416 (1986).

13. O. Kaczmarek et al., Prog. Theor. Phys. Suppl. 153, 287 (2004).

14. P. Petreczky and K. Petrov, Phys. Rev. D70, 054503 (2004).

15. O. Kaczmarek et al., Nucl. Phys. Proc. Suppl. 129, 560 (2004).

16. O. Kaczmarek et al., Phys. Rev. D70, 074505 (2004).

17. O. Kaczmarek and F. Zantow, hep-lat/0503017.

18. O. Kaczmarek and F. Zantow, to be published.

19. C. R. Allton et al., Phys. Rev. D66, 074507 (2002).

20. C. R. Allton et al., Phys. Rev. D68, 014507 (2003).

21. F. Karsch, E. Laermann, and A. Peikert, Nucl. Phys. B605, 579 (2001).

22. O. Kaczmarek et al., Phys. Rev. D62, 034021 (2000).

23. C. DeTar et al., Phys. Rev. D59, 031501 (1999).

24. S. Nadkarni, Phys. Rev. D33, 3738 (1986).

25. O. Philipsen, Phys. Lett. B535, 138 (2002).

26. O. Kaczmarek and F. Zantow, these proceedings, heplat/0502012.

27. UKQCD, P. Pennanen and C. Michael, hep-lat/0001015.

28. A. Dumitru et al., Phys. Rev. D70, 034511 (2004).

29. F. Zantow, hep-lat/0301014.

30. F. Karsch, these proceedings, hep-lat/0502014.

31. E. Gava and R. Jengo, Phys. Lett. B105, 285 (1981).

32. O. Kaczmarek, P. Petreczky, and F. Zantow, in preparation.

33. S. Datta et al., Phys. Rev. D69, 094507 (2004).

34. M. Asakawa and T. Hatsuda, Phys. Rev. Lett. 92, 012001 (2004).

35. M. Asakawa, T. Hatsuda, and Y. Nakahara, Nucl. Phys. A715, 863 (2003). 